\title{
CMS Line in Carrot
}

\section{Chetna Chugh $^{1 *}$, Sheshnath Mishra², Manisha Mangal ${ }^{3}$, Shrawan Singh $^{3}$ and Pritam Kalia ${ }^{3}$}

\author{
${ }^{1}$ Division of Biochemistry, ICAR-Indian Agricultural Research Institute, \\ New Delhi-110012, India \\ ${ }^{2}$ Shri Vaishnav Institute of Agriculture Science, Shri Vaishnav Vidyapeeth Vishwavidyalaya, \\ Indore-453111, India \\ ${ }^{5}$ Division of Vegetable Science, ICAR-Indian Agricultural Research Institute, \\ New Delhi-110012, India \\ *Corresponding author
}

\section{A B S T R A C T}

\begin{tabular}{|l|}
\hline K e y w o r d s \\
Hybrid carrot seed, \\
Cytoplosmic male \\
sterility, Maintainer, \\
Molecular Markers, \\
PCR. \\
\hline Article Info \\
\hline Accepted: \\
05 January 2020 \\
Available Online: \\
10 February 2020 \\
\hline
\end{tabular}

Male sterility in vegetables is commercially utilized to develop their hybrids due to cost effective and easy mechanism to produce large-scale hybrid seeds commercially. Different types of male sterility, and three-line and two-line hybrid breeding approaches involving Cytoplasmic male stesility (CMS) and environmental sensitive genic male sterile (GMS) lines, are used for hybrid seed production. In carrot which produces many but small sized flowers, making hand-emasculation tedious and time consuming. Identification of male sterile and fertile counterpart of CMS lines is also not possible at early seedling stage by using test cross methods. Marker assisted selection for male sterile and fertile (maintainer) genes greatly enhance selection of A and B plants in breeding program. So, molecular markers that can distinguish CMS status and fertile plants at an early seedling stage will be valuable tool in carrot breeding including CMS based hybrid seed programs in hybrid seed purity evaluation, selection and management. Keeping these in views, the present study was undertaken to characterize different sources of cytoplasmic male sterile lines and their maintainers. By using available molecular markers closely linked to sterility gene to distinguish our male sterile and fertile lines.

\section{Introduction}

Cytoplasmic male sterility (CMS) is a maternally inherited trait that has been correlated with changes of the mitochondrial genome or with incompatibility between nuclear and mitochondrial genomes. Evaluation of cytoplasm that induces CMS in carrot for use in breeding programs. Several induce 'brown anther' (Sa) male sterility in which the anthers are formed .Sa CMS cytoplasms have been isolated from several open pollinated varieties as well as from wild populations (Welch \& Grimball, 1947; Banga et al., 1964; Ronfort et al., 1995). 
Other CMS type in carrot is 'petaloid' (Sp), in which the stamens are replaced by petals or bractlike structures. This type of cytoplasm found in feral populations of (Daucus carota) ssp. carota from North America and Sweden. Petaloid male-sterile plants were found among wild carrots from USA, Canada, Sweden and Germany. The American sources of sterility were subsequently introduced into the cultivated germplasm allowing production of the modern hybrid varieties (reviewed in Bach 2000). Stamen petaloidy in cultivated carrots was also induced upon cytoplasm transfer from D. c. maritimus (Nothnagel et al., 1997).

CMS, caused by mutations in the mitochondrial genome (Jing et al., 2015), is found in higher plants and increases heterosis and improve genetic resources. Mitochondrial markers can be used to differentiate between the various types of CMS sources (Zhao et al., 2010; Wang et al., 2012; Zhang et al., 2013). In protoplast fusion (Yarrow et al., 1990) and distant hybridization (Jing et al., 2015) phenomenon of cytoplasmic male sterility (CMS; Kaul, 1988) a thesaurus contribution of extra-nuclear genes in flower formation. CMS plants are unable to produce fertile pollen and hence are male sterile. The maternal inheritance of the CMS indicates that the genetic information of either the mitochondria or the plastids participates in certain steps of flower formation. Mitochondrial genes have been recognized as being implicated in all well-investigated CMS systems.

In many cases of CMS, rearrangements in the mitochondrial genome were found to produce unique chimeric genes, and often function of genes encoding subunits of the mitochondrial ATP synthase is impaired, suggesting that reduced mitochondrial respiration and/or ATP production lead to male sterility (reviewed by Budar and Pelletier, 2001; Mackenzie and
McIntosh, 1999; Schnable and Wise, 1998). The mitochondrial dysfunction of CMS plants can be either maintained or concealed by specific nuclear gene functions leading, in the latter case, to restored male fertility. Genetic and molecular mechanisms of restoration vary among the different CMS systems (reviewed by Schnable and Wise, 1998). Elimination of CMS-related mitochondrial DNA segments (Janska et al., 1998), restoration of effective editing of specific mitochondrial mRNAs (Howad and Kempken, 1997) as well as posttranscriptional decrease of aberrant mitochondrial gene products (e.g. Gagliardi and Leaver, 1999; Menassa et al., 1999) and compensatory effects at the metabolic level (Cui et al., 1996; Liu et al., 2001) have been described. Tissuespecific differences in mitochondrial gene expression and in energy load are suggested explanations for the specific effects of mitochondrial dysfunction on male flower organs and pollen formation, respectively (e.g. Bergman et al., 2000; Conley and Hanson, 1995; Ducos et al., 2001). therefore the mitochondria have to impede with the function of certain, yet unrevealed nuclear genes during flower formation. Nuclear genome responsible for flower formation in plants. cytoplasmic male sterility indicates (CMS; Kaul, 1988) a participation of extra-nuclear genes in flower formation Inability of CMS plants produce fertile pollen and hence are male sterile. The maternal inheritance of the CMS trait indicates that the genetic information of either the mitochondria or the plastids participates in certain steps of flower formation. Mitochondrial genes have been identified as being involved in all well-investigated CMS systems. In many cases of CMS, rearrangements in the mitochondrial genome were found to create unique chimeric genes, and often function of genes encoding subunits of the mitochondrial ATP synthase is impaired, suggesting that reduced mitochondrial respiration and/or ATP 
production lead to male sterility (reviewed by Budar and Pelletier, 2001; Mackenzie and McIntosh, 1999; Schnable and Wise, 1998). The mitochondrial dysfunction of CMS plants can be either maintained or censored by specific nuclear gene functions leading, in the latter case, to restored male fertility. Genetic and molecular mechanisms of restoration vary among the different CMS systems (reviewed by Schnable and Wise, 1998). Elimination of CMS-related mitochondrial DNA segments (Janska et al., 1998), restoration of effective editing of specific mitochondrial mRNAs (Howad and Kempken, 1997) as well as posttranscriptional decrease of aberrant mitochondrial gene products (e.g. Gagliardi and Leaver, 1999; Menassa et al., 1999) and compensatory effects at the metabolic level (Cui et al., 1996; Liu et al., 2001) have been described.

Tissue-specific differences in mitochondrial gene expression and in energy demands are recommended explanations for the specific effects of mitochondrial dysfunction on male flower organs and pollen formation, respectively (e.g. Bergman et al., 2000; Conley and Hanson, 1995; Ducos et al., 2001). Consequently, the mitochondria have to impede with the function of certain, yet unknown nuclear genes during flower formation. impairement in CMS plants in late stages of pollen formation without affecting female organs or general flower architecture. But rarely, like in the 'petaloid' CMS system of Daucus carota (Dc) (Kitagawa et al., 1994; Linke et al., 1999; Nothnagel et al., 2000), is the whole composition of flowers altered, frequently showing homeotic-like organ replacements. similar floral malformations occur in alloplasmic lines of Nicotiana (Farbos et al., 2001; Kofer et al., 1991) and wheat (Murai et al., 2002) and in cybrids between Nicotiana and Hyoscyamus (Zubko et al., 2001). Exhibition of altered morphologyin CMS flowers of petals and stamens (Eisa and Wallace, 1969; Straub, 1971). Petals have sepaloid features (Kitagawa et al., 1994) and stamens are converted into either petals ('petaloid'-type) or carpel-like structures ('carpeloid'-type), respectively. Characterisation of petaloid CMS system of carrot at the genetic level (Banga et al., 1964; Börner et al., 1995; Thompson, 1961; Welch and Grimball, 1947; Wolyn and Chahal, 1998).

A single dominant gene (M) and two homozygous recessive alleles $(11, \mathrm{tt})$ are anticipated to maintain male sterility. Heterozygoty of the dominant allele (Mm) and a dominant allele $\mathrm{L}$ or $\mathrm{T}$ can re-establish partial fertility at high temperatures (Börner et al., 1995 and references therein). Molecular analysis of the mitochondrial DNA and mRNA exposed differences between fertile and male-sterile plants including rearrangements of genes as potential causes for the CMS phenotypes (Börner et al., 1995; Nakajima et al., 2001; Scheike et al., 1992; Szklarczyk et al., 2000).

Investigation of nuclear mutants of Antirrhinum and Arabidopsis reveals Similar homeotic organ alterations affecting two neighbouring flower whorls (Bowman et al., 1989; Schwarz-Sommer et al., 1990).observation of CMS phenotypes in carrot lines containing cytoplasms from Daucus carota subspecies (Linke et al., 1999) but these cytoplasms have not yet been integrated in to cultivated carrots for hybrid breeding. Brown anther CMS was the first type used for developing hybrid carrots, but the majority of current hybrid carrot varieties utilize petaloid cytoplasms derived from two different sources of Sp cytoplasm, designated 'Cornell' (SpC; Thompson, 1961) and 'Wisconsin Wild' (SpW; Morelock et al., 1996). The SpC and SpW cytoplasms were found in Massachusetts and Wisconsin, respectively. evaluation of $\mathrm{SpC}, \mathrm{SpW}$ and 
Canadian Sp cytoplasms with the cytoplasms of maintainer lines ( $\mathrm{N}$ cytoplasms) using mitochondrial restriction length fragment polymorphisms

(RFLPs) exposed that the Sp cytoplasms were very associated to each other and clearly distinct from $\mathrm{N}$ cytoplasms, which were found to be substantially more erratic (Bowes \& Wolyn, 1998; Pingitore et al., 1989; Scheike et al., 1992; Steinborn et al., 1992). A chloroplast RFLP marker has been acknowledged among $\mathrm{N}$ cytoplasms of cultivated carrot (Vivek et al., 1999), but chloroplast markers that differentiate $\mathrm{N}$ from $\mathrm{Sa}$ and Sp cytoplasms have not been found (Scheike et al., 1992; Vivek \& Simon, 1999).

Molecular markers that can envisage the CMS status at an early developmental stage will be essential tools in carrot breeding and seed making programs, as well as for basic studies of male sterility. Recently, six mitochondriaspecific Sequence Tagged Site (STS) primer pairs derived from Randomly Amplified Polymorphic DNA (RAPD) markers were reported to distinguish $\mathrm{SpC}$ and $\mathrm{SpW}$ cytoplasms from a collection of three male fertile inbred carrot lines and five open pollinated varieties (Nakajima et al., 1999).

However, only two of these primer pairs amplified fragments that were constantly linked with either $\mathrm{Sp}$ or $\mathrm{N}$ cytoplasms. Because of the high degree of disparity surrounded by mitochondrial genomes of fertile carrots and the energetic nature of the mitochondrial genome of higher plants in general, several markers may be requisite to establish whether an uncharacterized cytoplasm confers petaloidy.

In addition, a range of mitochondria-specific markers will be useful to make out the mitochondrial genomes of putative cybrids consequential from protoplast fusions, and to illustrate a wide range of wild and cultivated carrot germplasm.

Carrots instead of petaloid type of CMS are not exceptional among plants. Similar abnormalities have been reported for a few other genera like Nicotiana, Brassica and Plantago. Most cases of CMS in tobacco resulted from interspecific hybridization and recurrent backcrossing placing Nicotiana tabacum nuclear genome in the perspective of cytoplasm from some wild Nicotiana species (Kaul 1988; Bonnett et al., 1991).

Mutations in definite nuclear genes may also condition the conversion of stamens into petals. Agamous - floral homeotic mutant of Arabidopsis - and plena - its equivalent from Antirrhinum - are by far the best studied instances of this phenomenon (Causier et al., 2010).

According to the $\mathrm{ABC}(\mathrm{DE})$ model of floral morphogenesis (Ferrario et al., 2004), both mutants correspond to impairment of the socalled $\mathrm{C}$ function and its replacement with activity of genes representing A function. Joint actions of the $\mathrm{A}, \mathrm{B}$ and $\mathrm{E}$ functions indicate petal distinctiveness. Phenotypic similarities between petaloid CMS and nuclear homeotic mutations propose that the cytoplasm, by an as yet unknown mechanism, influences the expression of transcription factors controlling floral patterning (Linke $e t$ al., 2003).

It seems that cytoplasmic factors could act either through impairment of the $\mathrm{C}$ function or by enhancing the functions engaged in petal formation. In favor of the latter possibility, the combined ectopic expression of $A P 3, P I$ (both are $\mathrm{B}$ function genes) and $A P 1$ (A function) as well as $A P 3, P I$ together with SEP3 (E function) converts vegetative leaves into petaloid organs (Honma and Goto 2001). 


\section{Basic steps in the use of $\mathrm{cms}$}

A major role played by three in the use of CMS system. female parent represented as A line with good floral and agronomic traits, It is mostly a line of preferred genetic background (genetically Srfrf) where s stands for sterile cytoplasm and $\mathrm{rf}$ for a recessive male sterility gene. To maintain A line, an isogenic line with the genetic background of A line known as B line (maintainer), is required. To employ this system for hybrid seed production, the third line commonly known as $\mathrm{C}$ line (male parent) is there which also acts as the paramount specific combiner with A-line. Genetically this could be N/SRf/- (fertility restoration) or Nrfrf since the economic product in cauliflower is not the exact seed. To carry out additional hybridization workprotection of $\mathrm{A}, \mathrm{B}$ and $\mathrm{C}$ line is the then important step. For hybrid seed creation, female to male ratios generally favored are $2: 1 ; 3: 1$ or $4: 1$ depending on varietal characters.

\section{Mechanism of cms}

The occurrence of male sterility in wild species is revealed by the coexistence of hermaphrodite and female (male sterile) plants in natural populations (gynodioecy). Most of the studies on such populations have led to the emergence of theoretical models based on the premise that CMS is a result of interaction between nuclear and cytoplasmic genomes. Gynodioecy is supposed to appear and be maintained in populations either due to female advantage or counter selection of nuclear fertility restorers (Budar et al., 2003).

The genomic conflict theory (Cosmides and Tooby, 1981) provides for the interaction of cytoplasmic determinants (that prevent pollen production) and nuclear restorers (that restore fertility). The modes of inheritance of cytoplasmic (uniparental) and nuclear (biparental) factors generate a situation where selective interests are conflicting, resulting in differential resource allocation for pollen and seed production. Most of the CMS's were a result of an alloplasmic state, wherein an impaired mitochondrial activity occurred due to a conflict at genomic, proteomic or transcriptomic levels (Kaul, 1988).

Studies on the genetic and physiological factors underlying male sterility have revealed that significant differences exist between normal and sterile anthers (Nair, 1993). The tapetum, the innermost cell layer surrounding pollen grain, is a physiologically active site and nourishes the developing gametes. However, it degenerates later on and is not found as an organized tissue in mature anthers. The tapetum and other surrounding cell layers suffer early degeneration in sterile pollen as compared to fertile ones. An important question was: how does a mitochondrial dysfunction at the genetic and physiological level specifically alter pollen development (Levings, 1993).

Since mitochondria harbor genes governing vital biochemical pathways such as the electron transport system and encode enzyme systems and structural RNA's, how do such mutations specifically block pollen development, as mutations to any of them could be lethal? One explanation could be that mitochondrial genes are expressed differently during anther development. Such differential gene expression may occur either by the interaction of CMS genes with some unknown factors in target tissue leading to localized developmental changes or interference of CMS gene products in the physiological machinery resulting in reduced respiration and ATP synthesis (Levings, 1993).

Wallace (1989) suggested that minor impairments in mitochondrial function might 
selectively affect degeneration of specific cell types. Such deviations in expression were observed in all cell and tissue types and have also been reported in case of human cardiac dysrythmia (Wallace, 1988). Lee and Warmke (1979) observed a 10-fold increase in mitochondria/cell count in the tapetum during anther development. Such amplification indicates greater demand for energy

Even though male sterility is strongly associated with gene dysfunction in mitochondria, chloroplasts have emerged as a more than ideal choice for engineering male sterility through the transgenic approach. Such an approach offers a number of advantages such as high levels of transgene expression, multi-gene cassette engineering, transgene containment viz., maternal inheritance, lack of gene silencing, position effect due to sitespecific transgene integration, and lack of pleiotropic effects (Daniell, 2005).

Moreover, such engineering strategy can enable the transfer of male sterility systems in certain recalcitrant crops such as cotton and soybean (Grevich and Daniell, 2005). It also overcomes the disadvantages of nuclear transformation, wherein the transformants segregate for fertility and sterility (Ruiz and Daniell, 2005).

\section{Plant mitochondrial mutations}

Variation in the mitochondrial genome of plant and animal of variable sizes to undergo recombination (Arrieta-Montiel and Mackenzie, 2010). this recombination activity in plants can subdivide the genome to a highly redundant, multi-partite structure, with different submolecules maintain at varying levels. Sites with the potential to undergo recombination can be numerous within the mitochondrial genome, with 47 repeat pairs functionally identified in the Arabidopsis mitochondrial genome (Arrieta-Montiel et al., 2009, Davila et al., 2011 ). This type of recombination provides an opportunity for accelerate genome evolution and capacity for extensive genomic variation.

Variation of different forms are particularly prevalent in the mitochondrial genomes of plants, sequence chimeras from nonhomologous end joining, and express intermolecular genomic copy number changes within the mitochondrial population, formed by recombination and termed substoichiometric shifting (SSS). mutually phenomena are key components of CMS systems.

Identification of all CMS mitochondrial mutations comprise of sequence chimeras, generally fusing identifiable mitochondrial gene sequences with segments of unknown origin (Schnable and Wise, 1998, Hanson and Bentolila, 2004)., no two CMS mutations have been the same. rearrangement of these sequences give rise to new gene products that usually show some degree of hydrophobicity and their intraction with the mitochondrial membrane. various CMS sequences contain segments of genes involved in the ATPase compleximply that energy-associated alterations might participate in the CMS phenotype.

CMS mutations differ not only in their sequence origins, but often in their phenotype, from alterations in microspore development (Datta et al., 2002), to callose dissolution (Abad et al., 1995), to premature tapetal breakdown patterns (González-Melendi et al., 2008).

Whether these phenotypic differences are the consequence of distinct mechanisms of action or simply a reflection of variation in spatial and temporal expression patterns of the sterility lesion is not known. 


\section{Fertility restoration}

suppression of male sterile phenotype by nuclear fertility restorer genes. Identification of valuable genes provide insight into the nature of mitochondrial gene regulation (Schmitz-Linneweber and Small, 2008). majority of cloned genes are fertility restorer genes them have been found to contain pentatricopeptide repeats (PPR) within the protein. participation of PPR proteins in mRNA splicing, editing, and translation initiation processes (Delannoy et al., 2007). this family of genes functions in both mitochondria and plastids and is, numbering over 400 in Arabidopsis (Small and Peeters, 2000). even if essential for the proper processing and translation of native organellar transcripts, PPR-mediated processing can serve to inactivate a rearranged, CMSassociated gene transcript.

Interestingly, regions of a genome may find PPR protein-coding genes organized in large duplicated gene clusters, representing fertility restorer loci (Barr and Fishman, 2010). association of these genes within plant genomes suggests that they are subject to extreme evolutionary selection, somewhat similar in pattern to disease-resistance genes (Geddy and Brown, 2007).

In naturally occurring CMS hybrid inappropriateness systems, where the male sterility phenotype can arise by particular hybridizations, evidence from PPR protein gene organization and maintenance across angiosperms recommend co-evolution of these nuclear and cytoplasmic factors to influence cross compatibility.

Two other types of nuclear fertility restorers have also been reported. The first is the $R f 2$ restorer of maize CMS-T cytoplasm, which encodes an aldehyde dehydrogenase that is recommended to play a detoxification role
(Cui et al., 1996, Liu et al., 2001). a different type of nuclear restorer was reported in the common bean $F r$ gene, which effects thorough stoichiometric changes in mitochondrial genome organization and results in remarkable decrease of the mitochondrial male sterility sequence, $p v s$ orf239, to near imperceptible levels (Mackenzie and Chase, 1990). This latter fertility restoring mechanism is non-reversible in subsequent generations; therefore, it does not fall under the formal definition of a fertility restorer gene. However, such nucleardirected influences on mitochondrial genome organization likely play an vital role in controlling mitochondrial genetic variation in natural plant populations.

\section{Steadiness of the CMS trait}

An fundamental feature of the CMS mutation for use in crop breeding is its stability, with little or no spontaneous reversion to fertility. degeneration to fertility is linked with SSS activity (Arrieta-Montiel and Mackenzie, 2010).

Recombination-mediated genomic changing can result in rapid copy number decrease of the DNA molecule on which the CMS mutation resides, departure the remainder of the mitochondrial genome essentially unchanged (Janska et al., 1998, Bellaoui et al., 1998).

In this manner, the CMS mutation is silenced and the plant displays a male fertile phenotype. Reversion frequency in most CMS systems that have been studied appears to be influenced by nuclear genetic background (Mackenzie and McIntosh, 1999).

Therefore, a breeder wishing to employ a CMS system must test the strength of the system on a range of germplasm in the program. 
Male sterility: Metabolic and evolutionary implications

Observation of CMS in over 150 plant species (Kaul, 1988), signifying that the competence to convert to male sterility is established in plants. In CMS common bean (Arrieta-Montiel et al., 2001) and Brassica spp. (Murayama et al., 2004, Giancola et al., 2007), fertile plants from wild populations containS the male sterility gene at substoichiometric levels.

These observations imply that it is feasible to interconvert male fertile and sterile types under particular, not yet defined conditions. identification of two nuclear genes that control the mitochondrial SSS process, MSH1 (Abdelnoor et al., 2003) and RECA3 (Shedge et al., 2007). Disruption or RNAi suppression of the nuclear gene MSH1 results in a marked increase in the frequency of mitochondrial recombination (Sandhu $e t$ al., 2007) and, interestingly, results in a CMS phenotype in six different plant species tested to date (Shedge et al., 2010; Sandhu and Mackenzie, unpublished).

This raises the intriguing possibility that CMS induction in nature can occur under conditions that suppress MSH1 expression. Interestingly, transcription of MSH1 is markedly downregulated under environmental stresses, including heat, drought, cold, and high light (Zimmermann et al., 2004, Shedge et al., 2010).

Occurance of male sterility by numerous mechanisms in plants, the phenotype is apparently adaptive within a plant population under certain circumstances Male sterility has been linked with increases in seed production and vigor as the consequences of out-crossing (Charlesworth, 2002). The occurrence of gynodioecy, or the mixture within a plant population of both hermaphrodites and females (male steriles), has been expected to occur in over $7 \%$ of all plant species (McCauley and Olson, 2008).

In various of these cases, the male sterility appears to be cytoplasmic, likely a outcome of the dynamic nature of the mitochondrial genome. Important questions enduring in this field turn around the nature of environmental and/or cellular cues that generate interconversion of male fertile and male sterile phenotypes in natural populations and in agricultural field conditions.

\section{Organelle metabolism influences pollen development}

There is support to propose that organelle metabolism, and overall cellular metabolic state, influences pollen development. Mutation of the plastidial glyceraldehyde-3phosphate dehydrogenase, in the form of a gapcp1 gapcp2 double mutant, renders the plant male sterile (Muñoz-Bertomeu et al., 2010). alterations in pollen mutant displays unusual collapsd shapes due to the tapetum and possibly changes in carbohydrate pools and/or trehalose level.similarly, interruption of cytosolic phosphoglucomutase prevents both male and female gametogenesis, signifying that proper carbohydrate allotment is critical at these stages (Egli et al., 2010).

Temperature has a major communicate with on pollen development and viability. Cold temperature stress can hamper pollen development, and male-gametophytespecific classes of transcription factors appear to influence pollen response to adverse temperature effects (Zou et al., 2010).

The programming of tapteum for an apoptosis-like development (Wang et al., 
1999, Wu and Cheung, 2000, Love et al., 2008). tapetal degeneration is vital for this programmed pollen development (Parish and $\mathrm{Li}, 2010)$. It remains largely speculation whether mitochondrial cues signal this PCD response, but CMS system indicates that mitochondria manipulate this process (Chase, 2007). interference of the process can provide a means of engineering male sterility.

\section{Genetic Engineering of Male Sterility}

The availability of reliable male sterility in agricultural systems, useful in hybrid seed production, transgene containment, and decrease of overall airborne allergens. The deployment of transgenic crops that are male sterile provides greater promise that the transgenic genotype will remain restricted to the area in which it is planted, without fear for transport of pollen to adjoining fields. The plenty of biological detail presented for pollen pathways facilitates the engineering of transgenic approaches for male sterility induction, albeit with mixed success to date.

A number of anther-specific promoters are eagerly obtained from genes identified to specifically express during anther and pollen stages. As a recent example of this approach, five anther-specific promoters were isolated from Brassica oleracea and $B$. rapa and shown to convey in tapetal cells during meiocyte development.

Fusion of these promoters to candidate male sterility genes such as cysteine proteases BoCysP1 and BoCP3, and Gibberellininsensitive (gai) genes for beginning to Arabidopsis (Konagaya et al., 2008) created mixed results. appearance of BoCysP1 controlled by two specific promoters formed plants with complete male sterility.

Tapetal and middle layer cells of anthers expressing BoCysP1 showed swelling and vacuolation, perhaps intervention with the normal pattern of programmed cell death.

\section{Realization of male sterility in agricultural systems}

Genetic engineering attempts of male sterility, often useful in suppressing or disrupting normal pollen development patterns, have generally suffered from their infeasibility to scale up for agricultural implementation. An important motivation for identifying an effective genetic system for male sterility is the opportunity to cultivate hybrids. An $\mathrm{F}_{1}$ hybrid provides more uniform crop performance in the field and, more importantly, the significant advantage of heterosis.

Heterosis, or hybrid vigor, refers to the ability of the $F_{1}$ hybrid to outperform either of the parents used in the cross. Consequently, development of hybrids has, in the case of many crops, accounted for significant gains in overall crop yield. For example, hybrid rice in China can be credited with reducing the total amount of land planted to rice from 36 Mha in 1975 to 30 Mha in 2000, and increasing total production from 128 to 189 million tons, a yield increase of up to 6.2 tons/ha (http://www.fao.org/rice2004).

Several different genetic models proposed to report for heterosis, including dominance, overdominance, and pseudo-overdominance, all appearing to have some merit (Chen, 2010). Suggestion has been given on that epigenetic factors, influenced by circadiancontrolled regulatory mechanisms, may underlie much of the heterosis phenomenon.

However, what has not been investigated sufficiently is the influence of maternal effects on hybrid vigor. Often reciprocal 
differences in cross-compatibility and vigor outcomes will be observed in a cross. There are several cases in CMS plant crossed to its sterility maintainer line will create a more vital progeny than self-pollination of the identical maintainer line (S. Mackenzie, unpublished). In these crosses, the two hybridizing plants are almost indistinguishable in nuclear genotype, implying that the vigor may result from cytoplasmic factors. In fact, very little research has been conducted to inspect the possible authority of mitochondria or plastids, the two essential generators of ATP, on heterosis.

It is appealing to consider that organellar metabolic factors, together with epigenetic signals, may integrate to enhance energy in the hybrid. Organellar changes arising as a outcome of mutation or inhibition of MSH1 in Arabidopsis and other crop species give rise to remarkable changes in growth patterns and transcript profiles, suggesting that organellar functions can directly persuade several developmental pathways in plants (Shedge et al., 2007, Shedge et al., 2010, Sandhu et al., 2007; Sandhu and Mackenzie, unpublished).

The natural system of cytoplasmic male sterility remains the most generally utilized agriculturally. CMS has been effectively implemented in a number of crops, including rice, sorghum, millet, onion, sugar beet, and carrot, but restrictions exist with this genetic system as well. Following the Southern Corn Leaf Blight of 1970, when a huge area of the Midwestern United States was planted with CMS-T type maize that proved vulnerable to the T-toxin of Bipolaris maydis (Ullstrup, 1970), maize CMS is no longer used to such a great extent.

What was erudite from that episode was the need for multiple distinct, stable sources of CMS for any given crop to avoid overplanting to a single cytoplasm type. Furthermore, a useful source of CMS has not yet been identified in many crops in which hybrid vigor is well renowned. With the recent demonstration that CMS can be transgenically induced (Sandhu et al., 2007), the opportunity may now exist for detection of additional CMS lines. apparently, this type of cytoplasmic genetic manipulation can be carried out in various species and in several distinct genotypes. If MSH1 modulation occurs naturally in plant populations, and the CMS arising in these manipulations also occurs in nature, we can imagine that fertility restoration mechanisms are also relatively widespread

The accessibility of essential information on the genetic and physiological pathways variable stamen development provides welldocumented opportunities to persuade these processes transgenically. More practical strategies for introduction of male sterility traits to large-scale agricultural production are needed now.

\section{Advantages of cms over other systems}

In carrot, F1 hybrids are valuable due to uniform maturity, high early and total yield, better curd quality with respect to curd compression and colour as well as for resistance to insect-pests, diseases and adverse weather conditions An efficient, reliable and established method of F1 seed production without infectivity by selffertilized seeds from each parent is vital.

Manual emasculation and pollination method in carrot is not useful at commercial level due to incompatible flower size and structure. So far hybrids in carrot have been developed using self-incompatibility (SI) system. Several reports concerning 
selfincompatibility in Indian carrot which revealed that inbreds/lines of maturity group I have strongest self-incompatibility followed by maturity group II and group III. In the case of the snowball cauliflower group, self-incompatibility is either very weak or not present at all.

Moreover, SI system poses a risk of occurrence of selfing in hybrid seeds besides problems of maintenance, multiplication of SI lines through tedious bud pollination or $\mathrm{CO} 2$ and $\mathrm{NaCl}$ spray . and breaking down of SI under different environmental conditions like high temperature and drought .Even in case of genic male sterility (GMS), roguing of fertile individuals from the female line at the time of anthesis poses a main problem.

To overcome such problems, CMS system offers a better alternative. efficacy of CMSbased hybrids to exploit heterosis has been established by. In recent years, important heterosis has also been reported in hybrids developed using CMS system for yield, yield linked and quality traits

There are three types of male sterility in carrot:

Brown anther (ba) (Welch and Grimbal, 1947)

Petalloid (pt) (Thompson, 1962)

Gummifer (Nothnagel, 1992)

\section{Brown anther type}

The brown anther (ba) male sterility was first discovered in the cultivar Tendersweet and reported by Welch and Grimbal in 1947. Brown anther type male sterility is due to the interaction of "sa cytoplasm" with atleast two independent recessive nuclear genes. Expression of the brown anther sterility was due to a homozygous recessive locus Ms5 or a dominant allele for Ms4, but dominant allele of either of the two complimentary loci would restore the fertility.

\section{Petaloid male sterility}

Petaloid sterility is commercially used for hybrid seed production in the world. According to Morelock (1974), the pt type of male sterility is due to interaction between "Sp cytoplasm" and two independent dominant genes (M1 and M2). This is manifested as the replacement of stamens with petals (white petaloidy) or both stamens and petals with green bract like structures (green petaloidy) (Kitagawa, et al., 1994). It is stable across a wide range of environments through flowering and seed production. CMS system has been established for the first time in Asiatic carrot germplasm in India at IARI.

In India, at IARI, petaloid CGMS was transferred to nantes type and crossed it with the indigenous variety "Pusa yamdagini" to develop hybrid Pusa nayanjyoti in 2009. Cytoplasmic male sterility system has been established for the first time in tropical carrot and tropical carrot hybrid "Pusa Vasudha" has been developed which is the first from any public sector institution

\section{Mechanisms of male sterility}

\section{Cytological changes}

Breakdown in the process of microsporogenesis can occur at a pre-or postmeiotic stage. The abnormalities can involve aberration during the process of meiosis in the formation of tetrads, during the release of tetrad (the dissolution of callose), at the vacuolate microspore stage or at mature or near-mature pollen stage

\section{Biochemical changes}

Male sterility is accompanied by certain biochemical changes such as qualitative and 
quantitative changes in amino acids, protein, and enzymes in developing anther. It has been found to be associated with reduced levels of proline, leucine, isoleucine, phenylalanine and valine and increased levels of glycine, arginine and aspartic acids (Kaul, 1988). Level of proline has been found to be particularly affected. Kakihara et al., (1988) reported that mature male sterile anthers contain one-eighth amount of proline in comparison to the fertile anthers. Anthers of male sterile plants contain lower soluble protein content and fewer polypeptide bands. Some polypeptides synthesized in normal stamens were absent in mutant stamens. The activity of enzyme callase which is required for breakdown of callose that surrounds PMCs and the tetrad is decreased in male sterile anthers.

Low activity callase leads to premature or delayed release of meiocytes and microspore, resulting in male sterility. Male sterility is also accompanied by decreased activity of isozyme esterase which plays an important role in hydrolysis of sporopollenin polymer that is required for pollen formation.

Decreased activity of esterase in male sterile plants has been observed in tomato (Bhadula and Sawhney, 1987) and radish (Zhou and Zhang, 1994). Compared to fertile lines, there is reduced activity of amylase in male sterile anthers and it corresponds with high starch content and reduced levels of soluble sugars.

\section{References}

Abdelnoor R.V., Yule R., Elo A., Christensen A., Meyer-Gauen G., Mackenzie S.Substoichiometric shifting in the plant mitochondrial genome is influenced by a gene homologous to MutSProceedings of the National Academy of Sciences of the United States of America, 100 (2003), pp. 5968-5973

Arrieta-Montiel M., Lyznik A., Woloszynska M., Janska H., Tohme J., Mackenzie S.Tracing evolutionary and developmental implications of mitochondrial stoichiometric shifting in the common bean Genetics, 158 (2001), pp. 851-864

Arrieta-Montiel M., Mackenzie S.A.Plant Mitochondrial Genomes and RecombinationF. Kempken (Ed.), Advances in plant biology: Plant mitochondria, Springer Publ (2010), pp. 65-84

Arrieta-Montiel M.P., Shedge V., Davila J., Christensen A.C., Mackenzie S.A.Diversity of the Arabidopsis mitochondrial genome occurs via nuclear-controlled recombination activity Genetics, 183 (2009), pp. 1261-1268

Bach IC (2000) Molecular breeding of carrot (Daucus carota L.). PhD thesis, University of Wisconsin, Madison, WI, USA and The Royal Veterinary and Agricultural University, Frederiksberg, Denmark

Börner, T., Linke, B., Nothnagel, T., Scheike, R., Schulz, B., Steinborn, R., Brennicke, A., Stein, M. andWricke, G. ( 1995) Inheritance of nuclear and cytoplasmic factors affecting male sterility in Daucus carota. In Genetic Mechanisms for Hybrid Breeding ( U. Kück and G. Wricke, eds). Advances in Plant Breeding, Vol. 18. Berlin: Blackwell Science, pp. 111- 122.

Bowes, C.E. \& D.J. Wolyn, 1998. Phylogenetic relationships among fertile and petaloid male sterile accessions of carrot, Daucus carota L. Theor Appl Genet 96: 928-932.

Budar, F. and Pelletier, G. ( 2001) Male 
sterility in plants: occurrence, determinism, significance and use. C. R. Acad. Sci. III ( 324), 543-550.

Causier B, Schwarz-Sommer Z, Davies B. Floral organ identity: 20 years of ABCs. Sem Cell Dev Biol. 2010;21:73-79. doi: 10.1016/j.semcdb.2009.10.005.

Conley, C.A. and Hanson, M.R. ( 1995) How do alterations in plant mitochondrial genomes disrupt pollen development? J. Bioenerg. Biomembr. 27, 447-457.

Cui, X., Wise, R.P. and Schnable, P.S. ( 1996) The rf2 nuclear restorer gene of male-sterile T-cytoplasm maize. Science, 272, 1334- 1336.

Daniell, H. (2005). Engineering cytoplasmic male sterility via., chloroplast genome. ISB News Report.

Ducos, E., Touzet, P. and Boutry, M. ( 2001) The male sterile $G$ cytoplasm of wild beet displays modified mitochondrial respiratory complexes. Plant J. 26, 171- 180.

Farbos, I., Mouras, A., Bereterbide, A. and Glimelius, K. ( 2001) Defective cell proliferation in the floral meristem of alloplasmic plants of Nicotiana tabacum leads to abnormal floral organ development and male sterility. Plant J. 26, 131- 142.

Gagliardi, D. and Leaver, C.J. ( 1999) Polyadenylation accelerates the degradation of the mitochondrial mRNA associated with cytoplasmic male sterility in sunflower. EMBO J. 18, 3757- 3766.

Giancola S., Rao Y., Chaillou S., Hiard S., Martin-Canadell A., Pelletier G., et al.Cytoplasmic suppression of Ogura cytoplasmic male sterility in European natural populations of Raphanus raphanistrum Theoretical and Applied Genetics, 114 (2007), pp. 1333-1343
Grevich, J., Daniell, H. (2005). Chloroplast genetic engineering. Recent advances and perspectives. Critical Rev. Plant Sci. 24, 1-25.

Honma T, Goto K. Complexes of MADSbox proteins are sufficient to convert leaves into floral organs. Nature. 2001;409:525-529. doi: $10.1038 / 35054083$.

Howad, W. and Kempken, F. ( 1997) Cell type-specific loss of atp6 RNA editing in cytoplasmic male sterile Sorghum bicolor. Proc. Natl. Acad. Sci. USA, 94, 11090- 11095.

Janska, H., Sarria, R., Woloszynska, M., Arrieta-Montiel, M. and Mackenzie, S.A. ( 1998)Stoichiometric shifts in the common bean mitochondrial genome leading to male sterility and spontaneous reversion to fertility. Plant Cell, 10, 1163- 1180.

Jing Z. G., Pei X. L., Tang Z., Liu Q., Zhang X. L., Luo T. K., et al., (2015). Molecular identification of Ogu cytoplasmic male sterile and sequence analysis in broccoli (Brassica oleracea var. italica). Guihaia 35, 239-243. 10.11931/guihaia.gxzw201309005

Kakihara, F., Masahiro, K. and Tokumasu, S. 1988. Relationship between pollen degeneration and amino acids, especially proline, in male sterile Japanese radish (Raphanus sativus L. var. longipinnatus Bailey). Scintia Horticultare 36: 17-23.

Kaul, M.L.H. ( 1988) Male Sterility in Higher Plants. Heidelberg: SpringerVerlag.

Kitagawa, J. \& Posluszny, U., Gerrath, J. and Wolyn, J. D. 1994. Developmental and morphological analyses of homeotic cytoplasmic male sterile and fertile carrot flowers. Sexual Plant Reproduction. 7. 41-50 
Konagaya K., Ando S., Kamachi S., Tsuda M., Tabei Y.Efficient production of genetically engineered, male-sterile Arabidopsis thaliana using antherspecific promoters and genes derived from Brassica oleracea and $B$. rapaPlant Cell Reports, 27 (2008), pp. 1741-1754

Linke, B., T. Nothnagel \& T. Borner, 1999. Morphological characterization of modified flower morphology of three novel alloplasmic male sterile carrot sources. Plant Breeding 118: 543 548.

Mackenzie, S. and McIntosh, L. ( 1999) Higher plant mitochondria. Plant Cell, 11, 571- 585.

Menassa, R., L'Homme, Y. and Brown, G.G. ( 1999) Post-transcriptional and developmental regulation of a CMSassociated mitochondrial gene region by a nuclear restorer gene. Plant J. 17,491- 499 .

Morelock, T.E., P.W. Simon \& C.E. Peterson, 1996. Wisconsin Wild: Another petaloid male-sterile cytoplasm for carrot. HortSci 31: 887-888.

Muñoz-Bertomeu J., Cascales-Miñana B., Irles-Segura A., Mateu I., NunesNesi A., Fernie A.R., et al.The plastidial glyceraldehyde-3phosphate dehydrogenase is critical for viable pollen development in ArabidopsisPlant Physiology, 152 (2010), pp. 1830-1841

Murai, K., Takumi, S., Koga, H. and Ogihara, Y. ( 2002) Pistillody, homeotic transformation of stamens into pistil-like structures, caused by nuclear-cytoplasm interaction in wheat. Plant J. 29,169- 181.

Nakajima Y., T. Yamamoto, T. Muranaka \& K. Oeda, 1999. Genetic variation of petaloid male-sterile cytoplasm of carrot revealed by sequence tagged sites (STSs). Theor Appl Genet 99: 837-843.

Nakajima, Y., Yamamoto, T., Muranaka, T. and Oeda, K. ( 2001) A novel orfBrelated gene of carrot mitochondrial genomes that is associated with homeotic cytoplasmic male sterility (CMS). Plant Mol. Biol. 46, 99- 107.

Pingitore, M., B. Matthews \& J.P. Bottino, 1989. Analysis of the mitochondrial genome of Daucus carota with male sterile and male fertile cytoplasm. J Heredity 80: 143-145.

Ronfort, J., P. Saumitou-Laprade \& J. Cuguen, 1995. Mitochondrial DNA diversity and male sterility in natural populations of Daucus carota ssp carota. Theor Appl Genet 91: 150159

Sandhu A.S., Abdelnoor R.V., Mackenzie S.A.Transgenic induction of mitochondrial rearrangements for cytoplasmic male sterility in crop plantsProceedings of the National Academy of Sciences of the United States of America, 104 (2007), pp. 1766-1770

Scheike, R., E. Gerold, A. Brennicke, M. Mehring-Lemper \& G. Wricke, 1992. Unique patterns of mitochondrial genes, transcripts and proteins in different male-sterile cytoplasma of Daucus carota. Theor Appl Genet 83: 419-427

Shedge V., Arrieta-Montiel M., Christensen A.C., Mackenzie S.A.Plant mitochondrial recombination surveillance requires novel $\operatorname{Rec} A$ and MutS homologsPlant Cell, 19 (2007), pp. 1251-1264

Shedge V., Davila J., Arrieta-Montiel M.P., Mohammed S., Mackenzie S.A.Extensive rearrangement of the Arabidopsis mitochondrial genome elicits cellular conditions for thermotolerancePlant Physiology, 
152 (2010), pp. 1960-1970

Steinborn, R.A., A. Weihe \& T. Börner, 1992. Mitochondrial genome diversity within a cultivar of Daucus carota (ssp. sativus) revealed by restriction fragment analysis of single plants. Plant Breeding 109: 75-77.

Szklarczyk, M., Oczkowski, M., Augustyniak, H., Linke, B., Börner, T. and Michalik, B. ( 2000)Organisation and expression of mitochondrial atp 9 genes from CMS and fertile carrots. Theor. Appl. Genet. 100, 263-270.

Thompson, D.J., 1961. Studies on the inheritance of male-sterility in the carrot, Daucus carota L. var. sativa. Proc Amer Soc Hort Sci 78: 332 338.

Vivek, B.S., Q.A. Ngo \& P.W. Simon, 1999. Evidence for maternal inheritance of the chloroplast genome in cultivated carrot (Daucus carota L. ssp. sativus). Theor Appl Genet 98: 669-
672.

Wang Q. B., Zhang Y. Y., Fang Z. Y., Liu Y. M., Yang L. M., Zhuang M. (2012). Chloroplast and mitochondrial SSR help to distinguish allo-cytoplasmic male sterile types in cabbage (Brassica oleracea L. var. capitata). Mol. Breed. 30, 709-716. 10.1007/s11032-011-9656-9

Zhang R. J., Hu S. W., Yan J. Q., Sun G. L. (2013). Cytoplasmic diversity in Brassica rapa L. investigated by mitochondrial markers. Genet. Resour. Crop Evol. 60, 967-974. 10.1007/s10722-012-9892-9

Zhao H. X., Li Z. J., Hu S. W., Sun G. L., Chang J. J., Zhang Z. H. (2010). Identification of cytoplasm types in rapeseed (Brassica napus L) accessions by a multiplex PCR assay. Theor. Appl. Genet. 121, 643650. 10.1007/s00122-010-1336-3

\section{How to cite this article:}

Chetna Chugh, Sheshnath Mishra, Manisha Mangal, Shrawan Singh, Pritam Kalia. 2020. CMS Line in Carrot. Int.J.Curr.Microbiol.App.Sci. 9(02): 51-65. doi: https://doi.org/10.20546/ijcmas.2020.902.008 